On companion of Ostrowski inequality for mappings whose first derivatives absolute value are convex with applications

\author{
Mohammad W. Alomari, M. Emin Özdemir, and \\ Havva Kavurmac
}




\title{
ON COMPANION OF OSTROWSKI INEQUALITY FOR MAPPINGS WHOSE FIRST DERIVATIVES ABSOLUTE VALUE ARE CONVEX WITH APPLICATIONS
}

\author{
MOHAMMAD W. ALOMARI, M. EMIN ÖZDEMIR, AND HAVVA KAVURMAC
}

Received 21 February, 2012

\begin{abstract}
Several inequalities for a companion of Ostrowski inequality for absolutely continuous mappings whose first derivatives absolute value are convex (resp. concave) are established. Applications to a composite quadrature rule, to p.d.f.'s, and to special means are provided.
\end{abstract}

2000 Mathematics Subject Classification: 26D10; 26A15; 26A16; 26A51

Keywords: Convex mappings, Hermite-Hadamard inequality, Ostrowski inequality

\section{INTRODUCTION}

In 1938, Ostrowski established a very interesting inequality for differentiable mappings with bounded derivatives, as follows [8]:

Theorem 1. Let $f: I \subset \mathbb{R} \rightarrow \mathbb{R}$ be a differentiable mapping on $I^{\circ}$, interior of the interval $I$, such that $f^{\prime} \in L[a, b]$, where $a, b \in I$ with $a<b$. If $\left|f^{\prime}(x)\right| \leq M$, then the following inequality,

$$
\left|f(x)-\frac{1}{b-a} \int_{a}^{b} f(u) d u\right| \leq M(b-a)\left[\frac{1}{4}+\frac{\left(x-\frac{a+b}{2}\right)^{2}}{(b-a)^{2}}\right]
$$

holds for all $x \in[a, b]$. The constant $\frac{1}{4}$ is the best possible in the sense that it cannot be replaced by a smaller constant.

For recent results concerning Ostrowski inequality see [1], [2] and [3]. Also, the reader may be refer to the monograph [8] where various inequalities of Ostrowski type are discussed.

In [9], Guessab and Schmeisser have proved among others, the following companion of Ostrowski inequality: 
Theorem 2. Let $f:[a, b] \rightarrow \mathbb{R}$ satisfy the Lipschitz condition, i.e., $|f(t)-f(s)| \leq$ $M|t-s|$. Then for each $x \in\left[a, \frac{a+b}{2}\right]$, we have the inequality,

$$
\left|\frac{f(x)+f(a+b-x)}{2}-\frac{1}{b-a} \int_{a}^{b} f(t) d t\right| \leq\left[\frac{1}{8}+2\left(\frac{x-\frac{3 a+b}{4}}{b-a}\right)^{2}\right](b-a) M .
$$

The constant $1 / 8$ is the best possible in the sense that it cannot be replaced by a smaller constant.

We may also note that the best inequality in (1.1) is obtained for $x=\frac{3 a+b}{4}$, giving the trapezoid type inequality,

$$
\left|\frac{f\left(\frac{3 a+b}{4}\right)+f\left(\frac{a+3 b}{4}\right)}{2}-\frac{1}{b-a} \int_{a}^{b} f(t) d t\right| \leq \frac{(b-a)}{8} M
$$

The constant $1 / 8$ is sharp in (1.2) in the sense mentioned above.

Companions of Ostrowski integral inequality for absolutely continuous functions was considered by Dragomir in [6], pp.228, as follows :

Theorem 3. Let $f: I \subset \mathbb{R} \rightarrow \mathbb{R}$ be an absolutely continuous function on $[a, b]$. Then we have the inequalities,

$$
\begin{aligned}
& \left|\frac{f(x)+f(a+b-x)}{2}-\frac{1}{b-a} \int_{a}^{b} f(t) d t\right| \\
& \leq\left\{\begin{array}{l}
{\left[\frac{1}{8}+2\left(\frac{x-\frac{3 a+b}{4}}{b-a}\right)^{2}\right](b-a)\left\|f^{\prime}\right\|_{\infty}, \quad f^{\prime} \in L_{\infty}[a, b]} \\
\left(\frac{2}{q+1}\right)^{1 / q}\left[\left(\frac{x-a}{b-a}\right)^{q+1}+\left(\frac{\frac{a+b}{2}-x}{b-a}\right)^{q+1}\right]^{1 / q}(b-a)^{1 / q}\left\|f^{\prime}\right\|_{[a, b], p}, \\
{\left[\frac{1}{4}+\left|\frac{x-\frac{3 a+b}{b-a}}{b-a}\right|\right]\left\|f^{\prime}\right\|_{[a, b], 1}}
\end{array}\right.
\end{aligned}
$$

for all $x \in\left[a, \frac{a+b}{2}\right]$.

In [7], the following theorem which was obtained by Dragomir and Agarwal contains the Hermite-Hadamard type integral inequality: 
Theorem 4. Let $f: I^{\circ} \subseteq \mathbb{R} \rightarrow \mathbb{R}$ be a differentiable mapping on $I^{\circ}, a, b \in I^{\circ}$ with $a<b$. If $\left|f^{\prime}\right|$ is convex on $[a, b]$, then the following inequality holds:

$$
\left|\frac{f(a)+f(b)}{2}-\frac{1}{b-a} \int_{a}^{b} f(u) d u\right| \leq \frac{(b-a)\left(\left|f^{\prime}(a)\right|+\left|f^{\prime}(b)\right|\right)}{8} .
$$

In [5], S.S. Dragomir established some inequalities for this companion for mappings of bounded variation. Also, Z. Liu introduced some companions of an Ostrowski type integral inequality for functions whose derivatives are absolutely continuous in [10]. Recently, N.S. Barnett et al. have proved some companions for the Ostrowski inequality and the generalized trapezoid inequality in [4].

The aim of this paper is to study a companion of Ostrowski inequality Theorem 2 for the class of functions whose derivatives in absolute value are convex (concave) functions.

\section{RESULTS}

In order to prove our results, we need the following lemma (see [6]):

Lemma 1. Let $f: I \subset \mathbb{R} \rightarrow \mathbb{R}$ be an absolutely continuous mapping on $I^{\circ}$, where $a, b \in I$ with $a<b$, such that $f^{\prime} \in L_{1}[a, b]$. Then, the following equality holds

$$
\frac{f(x)+f(a+b-x)}{2}-\frac{1}{b-a} \int_{a}^{b} f(t) d t=\frac{1}{b-a} \int_{a}^{b} p(x, t) f^{\prime}(t) d t,
$$

where

$$
p(x, t)= \begin{cases}t-a, & t \in[a, x] \\ t-\frac{a+b}{2}, & t \in(x, a+b-x], \\ t-b, & t \in(a+b-x, b]\end{cases}
$$

for all $x \in\left[a, \frac{a+b}{2}\right]$.

A simple proof of the equality can be done by performing integration by parts. The details are left to the interested reader (see [6]).

Let us begin with the following result:

Theorem 5. Let $f: I \subset \mathbb{R} \rightarrow \mathbb{R}$ be an absolutely continuous mapping on $I^{\circ}$, where $a, b \in I$ with $a<b$, such that $f^{\prime} \in L_{1}[a, b]$. If $\left|f^{\prime}\right|$ is convex on $[a, b]$, then we have the following inequality:

$$
\left|\frac{f(x)+f(a+b-x)}{2}-\frac{1}{b-a} \int_{a}^{b} f(t) d t\right|
$$




$$
\begin{aligned}
\leq & \frac{(x-a)^{2}}{6(b-a)}\left(\left|f^{\prime}(a)\right|+\left|f^{\prime}(b)\right|\right) \\
& +\frac{8(x-a)^{2}+3(a+b-2 x)^{2}}{24(b-a)}\left(\left|f^{\prime}(x)\right|+\left|f^{\prime}(a+b-x)\right|\right)
\end{aligned}
$$

for all $x \in\left[a, \frac{a+b}{2}\right]$.

Proof. Using Lemma 1 and the modulus, we have

$$
\begin{aligned}
& \left|\frac{f(x)+f(a+b-x)}{2}-\frac{1}{b-a} \int_{a}^{b} f(t) d t\right| \\
& \leq \frac{1}{b-a} \int_{a}^{b}|p(x, t)|\left|f^{\prime}(t)\right| d t \\
& =\frac{1}{b-a}\left[\int_{a}^{x}|p(x, t)|\left|f^{\prime}(t)\right| d t+\int_{x}^{a+b-x}|p(x, t)|\left|f^{\prime}(t)\right| d t\right. \\
& \left.\quad+\int_{a+b-x}^{b}|p(x, t)|\left|f^{\prime}(t)\right| d t\right]
\end{aligned}
$$

Since $\left|f^{\prime}\right|$ is convex on $[a, b]=[a, x] \cup(x, a+b-x] \cup(a+b-x, b]$, therefore we have

$$
\begin{gathered}
\left|f^{\prime}(t)\right| \leq \frac{t-a}{x-a}\left|f^{\prime}(x)\right|+\frac{x-t}{x-a}\left|f^{\prime}(a)\right|, \quad t \in[a, x] \\
\left|f^{\prime}(t)\right| \leq \frac{t-x}{a+b-2 x}\left|f^{\prime}(a+b-x)\right|+\frac{a+b-x-t}{a+b-2 x}\left|f^{\prime}(x)\right|, \quad t \in(x, a+b-x] ;
\end{gathered}
$$

and

$$
\left|f^{\prime}(t)\right| \leq \frac{t-a-b+x}{x-a}\left|f^{\prime}(b)\right|+\frac{b-t}{x-a}\left|f^{\prime}(a+b-x)\right|, \quad t \in(a+b-x, b] ;
$$

which follows that,

$$
\begin{aligned}
& \left|\frac{f(x)+f(a+b-x)}{2}-\frac{1}{b-a} \int_{a}^{b} f(t) d t\right| \\
& \leq \frac{1}{b-a} \int_{a}^{x}|t-a|\left[\frac{t-a}{x-a}\left|f^{\prime}(x)\right|+\frac{x-t}{x-a}\left|f^{\prime}(a)\right|\right] d t \\
& +\frac{1}{b-a} \int_{x}^{a+b-x}\left|t-\frac{a+b}{2}\right|\left[\frac{t-x}{a+b-2 x}\left|f^{\prime}(a+b-x)\right|\right. \\
& \left.+\frac{a+b-x-t}{a+b-2 x}\left|f^{\prime}(x)\right|\right] d t \\
& +\frac{1}{b-a} \int_{a+b-x}^{b}|t-b|\left[\frac{t-a-b+x}{x-a}\left|f^{\prime}(b)\right|+\frac{b-t}{x-a}\left|f^{\prime}(a+b-x)\right|\right] d t
\end{aligned}
$$




$$
\begin{aligned}
= & \frac{1}{(b-a)(x-a)}\left[\left|f^{\prime}(x)\right| \frac{(x-a)^{3}}{3}+\left|f^{\prime}(a)\right| \frac{(x-a)^{3}}{6}\right] \\
& +\frac{1}{(b-a)(a+b-2 x)} \frac{(a+b-2 x)^{3}}{8}\left[\left|f^{\prime}(a+b-x)\right|+\left|f^{\prime}(x)\right|\right] \\
& +\frac{1}{(b-a)(x-a)}\left[\left|f^{\prime}(b)\right| \frac{(x-a)^{3}}{6}+\left|f^{\prime}(a+b-x)\right| \frac{(x-a)^{3}}{3}\right] \\
= & \frac{(x-a)^{2}}{6(b-a)}\left[\left|f^{\prime}(a)\right|+2\left|f^{\prime}(x)\right|+2\left|f^{\prime}(a+b-x)\right|+\left|f^{\prime}(b)\right|\right] \\
& +\frac{(a+b-2 x)^{2}}{8(b-a)}\left[\left|f^{\prime}(a+b-x)\right|+\left|f^{\prime}(x)\right|\right] \\
= & \frac{(x-a)^{2}}{6(b-a)}\left(\left|f^{\prime}(a)\right|+\left|f^{\prime}(b)\right|\right) \\
& +\frac{8(x-a)^{2}+3(a+b-2 x)^{2}}{24(b-a)}\left(\left|f^{\prime}(x)\right|+\left|f^{\prime}(a+b-x)\right|\right),
\end{aligned}
$$

where

$$
\int_{x}^{a+b-x}(t-x)\left|t-\frac{a+b}{2}\right| d t=\int_{x}^{a+b-x}(a+b-x-t)\left|t-\frac{a+b}{2}\right| d t=\frac{(a+b-2 x)^{3}}{8},
$$

which completes the proof.

An Ostrowski type inequality may be deduced as follows:

Corollary 1. Let $f$ as in Theorem 5. Additionally, if $f$ is symmetric about the $x$-axis, i.e., $f(a+b-x)=f(x)$, we have

$$
\begin{aligned}
& \left|f(x)-\frac{1}{b-a} \int_{a}^{b} f(t) d t\right| \\
\leq & \frac{(x-a)^{2}}{6(b-a)}\left(\left|f^{\prime}(a)\right|+\left|f^{\prime}(b)\right|\right) \\
& +\frac{8(x-a)^{2}+3(a+b-2 x)^{2}}{24(b-a)}\left(\left|f^{\prime}(x)\right|+\left|f^{\prime}(a+b-x)\right|\right)
\end{aligned}
$$

for all $x \in\left[a, \frac{a+b}{2}\right]$.

Remark 1. In Theorem 5, if we choose $x=a$, then we get

$$
\left|\frac{f(a)+f(b)}{2}-\frac{1}{b-a} \int_{a}^{b} f(t) d t\right| \leq \frac{b-a}{8}\left(\left|f^{\prime}(a)\right|+\left|f^{\prime}(b)\right|\right) .
$$

which is the inequality in (1.4). 
Corollary 2. In Theorem 5, if we choose

(1) $x=\frac{3 a+b}{4}$, then we get

$$
\begin{aligned}
& \left|\frac{1}{2}\left[f\left(\frac{3 a+b}{4}\right)+f\left(\frac{a+3 b}{4}\right)\right]-\frac{1}{b-a} \int_{a}^{b} f(t) d t\right| \\
\leq & \frac{(b-a)}{96}\left[\left|f^{\prime}(a)\right|+5\left|f^{\prime}\left(\frac{3 a+b}{4}\right)\right|+5\left|f^{\prime}\left(\frac{a+3 b}{4}\right)\right|+\left|f^{\prime}(b)\right|\right]
\end{aligned}
$$

(2) $x=\frac{a+b}{2}$, then we get

$$
\left|f\left(\frac{a+b}{2}\right)-\frac{1}{b-a} \int_{a}^{b} f(t) d t\right| \leq \frac{(b-a)}{24}\left[\left|f^{\prime}(a)\right|+4\left|f^{\prime}\left(\frac{a+b}{2}\right)\right|+\left|f^{\prime}(b)\right|\right] .
$$

Another result may be considered using the Hölder inequality, as follows:

Theorem 6. Let $f: I \subset \mathbb{R} \rightarrow \mathbb{R}$ be an absolutely continuous mapping on $I^{\circ}$, where $a, b \in I$ with $a<b$, such that $f^{\prime} \in L_{1}[a, b]$. If $\left|f^{\prime}\right|^{q}, q>1$ is convex on $[a, b]$, then we have the following inequality:

$$
\begin{aligned}
& \left|\frac{f(x)+f(a+b-x)}{2}-\frac{1}{b-a} \int_{a}^{b} f(t) d t\right| \\
& \leq \frac{1}{2^{1 / q}(b-a)(p+1)^{1 / p}}\left\{(x-a)^{2}\left[\left|f^{\prime}(a)\right|^{q}+\left|f^{\prime}(x)\right|^{q}\right]^{1 / q}\right. \\
& \quad+\frac{(a+b-2 x)^{2}}{2}\left[\left|f^{\prime}(x)\right|^{q}+\left|f^{\prime}(a+b-x)\right|^{q}\right]^{1 / q} \\
& \left.\quad+(x-a)^{2}\left[\left|f^{\prime}(a+b-x)\right|^{q}+\left|f^{\prime}(b)\right|^{q}\right]^{1 / q}\right\},
\end{aligned}
$$

for all $x \in\left[a, \frac{a+b}{2}\right]$, where $\frac{1}{p}+\frac{1}{q}=1$.

Proof. Using Lemma 1 and the Hölder inequality, we have

$$
\begin{aligned}
& \left|\frac{f(x)+f(a+b-x)}{2}-\frac{1}{b-a} \int_{a}^{b} f(t) d t\right| \\
& \leq \frac{1}{b-a} \int_{a}^{b}|p(x, t)|\left|f^{\prime}(t)\right| d t \\
& =\frac{1}{b-a}\left[\int_{a}^{x}|t-a|\left|f^{\prime}(t)\right| d t+\int_{x}^{a+b-x}\left|t-\frac{a+b}{2}\right|\left|f^{\prime}(t)\right| d t\right. \\
& \left.\quad+\int_{a+b-x}^{b}|t-b|\left|f^{\prime}(t)\right| d t\right]
\end{aligned}
$$




$$
\begin{aligned}
\leq & \frac{1}{b-a}\left[\left(\int_{a}^{x}|t-a|^{p} d t\right)^{1 / p}\left(\int_{a}^{x}\left|f^{\prime}(t)\right|^{q} d t\right)^{1 / q}\right. \\
& +\left(\int_{x}^{a+b-x}\left|t-\frac{a+b}{2}\right|^{p} d t\right)^{1 / p}\left(\int_{x}^{a+b-x}\left|f^{\prime}(t)\right|^{q} d t\right)^{1 / q} \\
& \left.+\left(\int_{a+b-x}^{b}|t-b|^{p} d t\right)^{1 / p}\left(\int_{a+b-x}^{b}\left|f^{\prime}(t)\right|^{q} d t\right)^{1 / q}\right]
\end{aligned}
$$

Since $\left|f^{\prime}\right|^{q}$ is convex on $[a, b]=[a, x] \cup(x, a+b-x] \cup(a+b-x, b]$, therefore we

have

$$
\left|f^{\prime}(t)\right|^{q} \leq \frac{t-a}{x-a}\left|f^{\prime}(x)\right|^{q}+\frac{x-t}{x-a}\left|f^{\prime}(a)\right|^{q}, t \in[a, x]
$$

$\left|f^{\prime}(t)\right|^{q} \leq \frac{t-x}{a+b-2 x}\left|f^{\prime}(a+b-x)\right|^{q}+\frac{a+b-x-t}{a+b-2 x}\left|f^{\prime}(x)\right|^{q}, t \in(x, a+b-x] ;$

and

$$
\left|f^{\prime}(t)\right|^{q} \leq \frac{t-a-b+x}{x-a}\left|f^{\prime}(b)\right|^{q}+\frac{b-t}{x-a}\left|f^{\prime}(a+b-x)\right|^{q}, t \in(a+b-x, b] ;
$$

which follows that,

$$
\begin{aligned}
& \left|\frac{f(x)+f(a+b-x)}{2}-\frac{1}{b-a} \int_{a}^{b} f(t) d t\right| \\
& \leq \frac{1}{b-a}\left[\left(\int_{a}^{x}|t-a|^{p} d t\right)^{1 / p}\left(\int_{a}^{x}\left[\frac{t-a}{x-a}\left|f^{\prime}(x)\right|^{q}+\frac{x-t}{x-a}\left|f^{\prime}(a)\right|^{q}\right] d t\right)^{1 / q}\right. \\
& \quad+\left(\int_{x}^{a+b-x}\left|t-\frac{a+b}{2}\right|^{p} d t\right)^{1 / p} \\
& \quad \times\left(\int_{x}^{a+b-x}\left[\frac{t-x}{a+b-2 x}\left|f^{\prime}(a+b-x)\right|^{q}+\frac{a+b-x-t}{a+b-2 x}\left|f^{\prime}(x)\right|^{q}\right] d t\right)^{1 / q} \\
& \quad+\left(\int_{a+b-x}^{b}|t-b|^{p} d t\right)^{1 / p} \\
& \left.\quad \times\left(\int_{a+b-x}^{b}\left[\frac{t-a-b+x}{x-a}\left|f^{\prime}(b)\right|^{q}+\frac{b-t}{x-a}\left|f^{\prime}(a+b-x)\right|^{q}\right] d t\right)^{1 / q}\right] \\
& \quad=\frac{1}{b-a}\left[\left(\frac{(x-a)^{p+1}}{(p+1)}\right)^{1 / p}\left(\frac{x-a}{2}\right)^{1 / q}\left(\left|f^{\prime}(a)\right|^{q}+\left|f^{\prime}(x)\right|^{q}\right)^{1 / q}\right.
\end{aligned}
$$




$$
\begin{aligned}
& +\left(\frac{2}{(p+1)}\left(\frac{a+b}{2}-x\right)^{p+1}\right)^{1 / p} \cdot \\
& +\left(\frac{a+b}{2}-x\right)^{1 / q}\left(\left|f^{\prime}(x)\right|^{q}+\left|f^{\prime}(a+b-x)\right|^{q}\right)^{1 / q} \\
& \left.\left.=\frac{(x-a)^{p+1}}{(p+1)}\right)^{1 / p}\left(\frac{x-a}{2}\right)^{1 / q}\left(\left|f^{\prime}(a+b-x)\right|^{q}+\left|f^{\prime}(b)\right|^{q}\right)^{1 / q}\right] \\
& +\frac{(a+b-2 x)^{2}}{2}\left(\left|f^{\prime}(x)\right|^{q}+\left|f^{\prime}(a+b-x)\right|^{q}\right)^{1 / q} \\
& \left.+(x-a)^{2}\left(\left|f^{\prime}(a+b-x)\right|^{q}+\left|f^{\prime}(b)\right|^{q}\right)^{1 / q}\right]
\end{aligned}
$$

since $\frac{1}{p}+\frac{1}{q}=1, q>1$, which completes the proof.

Corollary 3. Let $f$ as in Theorem 6. Additionally, if $f$ is symmetric about the $x$-axis, i.e., $f(a+b-x)=f(x)$, we have

$$
\begin{aligned}
\left|f(x)-\frac{1}{b-a} \int_{a}^{b} f(t) d t\right| \leq & \frac{1}{2^{1 / q}(b-a)(p+1)^{1 / p}} \\
& \times\left\{(x-a)^{2}\left[\left|f^{\prime}(a)\right|^{q}+\left|f^{\prime}(x)\right|^{q}\right]^{1 / q}\right. \\
& +\frac{(a+b-2 x)^{2}}{2}\left[\left|f^{\prime}(x)\right|^{q}+\left|f^{\prime}(a+b-x)\right|^{q}\right]^{1 / q} \\
& \left.+(x-a)^{2}\left[\left|f^{\prime}(a+b-x)\right|^{q}+\left|f^{\prime}(b)\right|^{q}\right]^{1 / q}\right\}
\end{aligned}
$$

for all $x \in\left[a, \frac{a+b}{2}\right]$.

Corollary 4. In Theorem 6, if we choose

(1) $x=a$, then we get

$$
\left|\frac{f(a)+f(b)}{2}-\frac{1}{b-a} \int_{a}^{b} f(t) d t\right| \leq \frac{(b-a)}{2^{1+\frac{1}{q}}(1+p)^{1 / p}}\left(\left|f^{\prime}(a)\right|^{q}+\left|f^{\prime}(b)\right|^{q}\right)^{1 / q} .
$$

(2) $x=\frac{3 a+b}{4}$, then we get

$$
\left|\frac{1}{2}\left[f\left(\frac{3 a+b}{4}\right)+f\left(\frac{a+3 b}{4}\right)\right]-\frac{1}{b-a} \int_{a}^{b} f(t) d t\right|
$$




$$
\begin{aligned}
\leq & \frac{(b-a)}{2^{4+\frac{1}{q}}(1+p)^{1 / p}}\left\{\left(\left|f^{\prime}(a)\right|^{q}+\left|f^{\prime}\left(\frac{3 a+b}{4}\right)\right|^{q}\right)^{1 / q}\right. \\
& +2\left(\left|f^{\prime}\left(\frac{3 a+b}{4}\right)\right|^{q}+\left|f^{\prime}\left(\frac{a+3 b}{4}\right)\right|^{q}\right)^{1 / q} \\
& \left.+\left(\left|f^{\prime}\left(\frac{a+3 b}{4}\right)\right|^{q}+\left|f^{\prime}(b)\right|^{q}\right)^{1 / q}\right\} .
\end{aligned}
$$

(3) $x=\frac{a+b}{2}$, then we get

$$
\begin{aligned}
& \left|f\left(\frac{a+b}{2}\right)-\frac{1}{b-a} \int_{a}^{b} f(t) d t\right| \\
& \leq \frac{(b-a)}{2^{2+\frac{1}{q}}(1+p)^{1 / p}}\left[\left(\left|f^{\prime}(a)\right|^{q}+\left|f^{\prime}\left(\frac{a+b}{2}\right)\right|^{q}\right)^{1 / q}\right. \\
& \left.\quad+\left(\left|f^{\prime}\left(\frac{a+b}{2}\right)\right|^{q}+\left|f^{\prime}(b)\right|^{q}\right)^{1 / q}\right] .
\end{aligned}
$$

The following result holds for concave mappings.

Theorem 7. Let $f: I \subset \mathbb{R} \rightarrow \mathbb{R}$ be an absolutely continuous mapping on $I^{\circ}$, where $a, b \in I$ with $a<b$, such that $f^{\prime} \in L_{1}[a, b]$. If $\left|f^{\prime}\right|^{q}, q>1$ is concave on $[a, b]$, then we have the following inequality:

$$
\begin{aligned}
& \left|\frac{f(x)+f(a+b-x)}{2}-\frac{1}{b-a} \int_{a}^{b} f(t) d t\right| \\
& \leq \frac{1}{(b-a)(1+p)^{1 / p}} \\
& \times\left[(x-a)^{2}\left(\left|f^{\prime}\left(\frac{a+x}{2}\right)\right|+\left|f^{\prime}\left(\frac{2 b+a-x}{2}\right)\right|\right)+\frac{(a+b-2 x)^{2}}{2}\left|f^{\prime}\left(\frac{a+b}{2}\right)\right|\right]
\end{aligned}
$$

for all $x \in\left[a, \frac{a+b}{2}\right]$, where $\frac{1}{p}+\frac{1}{q}=1$.

Proof. From Lemma 1, and by (2.5), we have

$$
\begin{aligned}
& \left|\frac{f(x)+f(a+b-x)}{2}-\frac{1}{b-a} \int_{a}^{b} f(t) d t\right| \\
& \leq \frac{1}{b-a}\left[\left(\int_{a}^{x}|t-a|^{p} d t\right)^{1 / p}\left(\int_{a}^{x}\left|f^{\prime}(t)\right|^{q} d t\right)^{1 / q}\right.
\end{aligned}
$$




$$
\begin{aligned}
& +\left(\int_{x}^{a+b-x}\left|t-\frac{a+b}{2}\right|^{p} d t\right)^{1 / p}\left(\int_{x}^{a+b-x}\left|f^{\prime}(t)\right|^{q} d t\right)^{1 / q} \\
& \left.+\left(\int_{a+b-x}^{b}|t-b|^{p} d t\right)^{1 / p}\left(\int_{a+b-x}^{b}\left|f^{\prime}(t)\right|^{q} d t\right)^{1 / q}\right] .
\end{aligned}
$$

Now, let us write,

$$
\begin{gathered}
\int_{a}^{x}\left|f^{\prime}(t)\right|^{q} d t=(x-a) \int_{0}^{1}\left|f^{\prime}(\lambda x+(1-\lambda) a)\right|^{q} d \lambda \\
\int_{x}^{a+b-x}\left|f^{\prime}(t)\right|^{q} d t=(a+b-2 x) \int_{0}^{1}\left|f^{\prime}(\lambda(a+b-x)+(1-\lambda) x)\right|^{q} d \lambda,
\end{gathered}
$$

and

$$
\int_{a+b-x}^{b}\left|f^{\prime}(t)\right|^{q} d t=(x-a) \int_{0}^{1}\left|f^{\prime}(\lambda b+(1-\lambda)(a+b-x))\right|^{q} d \lambda .
$$

Since $\left|f^{\prime}\right|^{q}, q>1$ is concave on $[a, b]=[a, x] \cup(x, a+b-x] \cup(a+b-x, b]$, we can use the Jensen integral inequality to obtain

$$
\begin{aligned}
& (x-a) \int_{0}^{1}\left|f^{\prime}(\lambda x+(1-\lambda) a)\right|^{q} d \lambda \\
& =(x-a) \int_{0}^{1} \lambda^{0}\left|f^{\prime}(\lambda x+(1-\lambda) a)\right|^{q} d \lambda \\
& \leq(x-a)\left(\int_{0}^{1} \lambda^{0} d \lambda\right)\left|f^{\prime}\left(\frac{1}{\int_{0}^{1} \lambda^{0} d \lambda} \int_{0}^{1}(\lambda x+(1-\lambda) a) d \lambda\right)\right|^{q} \\
& =(x-a)\left|f^{\prime}\left(\frac{x+a}{2}\right)\right|^{q}
\end{aligned}
$$

and analogously

$$
\begin{gathered}
(a+b-2 x) \int_{0}^{1}\left|f^{\prime}(\lambda(a+b-x)+(1-\lambda) x)\right|^{q} d \lambda \leq(a+b-2 x)\left|f^{\prime}\left(\frac{a+b}{2}\right)\right|^{q}, \\
(x-a) \int_{0}^{1}\left|f^{\prime}(\lambda b+(1-\lambda)(a+b-x))\right|^{q} d \lambda \leq(x-a)\left|f^{\prime}\left(\frac{2 b+a-x}{2}\right)\right|^{q} .
\end{gathered}
$$

Combining all above inequalities, we get

$$
\left|\frac{f(x)+f(a+b-x)}{2}-\frac{1}{b-a} \int_{a}^{b} f(t) d t\right|
$$




$$
\begin{aligned}
\leq & \frac{1}{b-a}\left[\left(\frac{(x-a)^{p+1}}{(p+1)}\right)^{1 / p}(x-a)^{1 / q}\left|f^{\prime}\left(\frac{x+a}{2}\right)\right|\right] \\
& +\left(\frac{2}{(p+1)}\left(\frac{a+b}{2}-x\right)^{p+1}\right)^{1 / p}(a+b-2 x)^{1 / q}\left|f^{\prime}\left(\frac{a+b}{2}\right)\right| \\
& +\left(\frac{(x-a)^{p+1}}{(p+1)}\right)^{1 / p}(x-a)^{1 / q}\left|f^{\prime}\left(\frac{2 b+a-x}{2}\right)\right| \\
= & \frac{1}{(b-a)(1+p)^{1 / p}} \\
\times & {\left[(x-a)^{2}\left(\left|f^{\prime}\left(\frac{a+x}{2}\right)\right|+\left|f^{\prime}\left(\frac{2 b+a-x}{2}\right)\right|\right)+\frac{(a+b-2 x)^{2}}{2}\left|f^{\prime}\left(\frac{a+b}{2}\right)\right|\right], }
\end{aligned}
$$

for all $x \in\left[a, \frac{a+b}{2}\right]$, where $\frac{1}{p}+\frac{1}{q}=1, q>1$, which is required.

Therefore, we may state the following Ostrowski type inequality:

Corollary 5. Let $f$ as in Theorem 7. Additionally, if $f$ is symmetric about the $x$-axis, i.e., $f(a+b-x)=f(x)$, we have

$$
\begin{aligned}
& \left|f(x)-\frac{1}{b-a} \int_{a}^{b} f(t) d t\right| \\
& \leq \frac{1}{(b-a)(1+p)^{1 / p}} \\
& \times\left[(x-a)^{2}\left(\left|f^{\prime}\left(\frac{a+x}{2}\right)\right|+\left|f^{\prime}\left(\frac{2 b+a-x}{2}\right)\right|\right)+\frac{(a+b-2 x)^{2}}{2}\left|f^{\prime}\left(\frac{a+b}{2}\right)\right|\right],
\end{aligned}
$$

for all $x \in\left[a, \frac{a+b}{2}\right]$.

Corollary 6. In Theorem 7 , if we choose

(1) $x=a$, then we get

$$
\left|\frac{f(a)+f(b)}{2}-\frac{1}{b-a} \int_{a}^{b} f(t) d t\right| \leq \frac{(b-a)}{2(1+p)^{1 / p}}\left|f^{\prime}\left(\frac{a+b}{2}\right)\right| .
$$

(2) $x=\frac{3 a+b}{4}$, then we get

$$
\left|\frac{1}{2}\left[f\left(\frac{3 a+b}{4}\right)+f\left(\frac{a+3 b}{4}\right)\right]-\frac{1}{b-a} \int_{a}^{b} f(t) d t\right|
$$


$\leq \frac{(b-a)}{16(1+p)^{1 / p}}\left[\left|f^{\prime}\left(\frac{7 a+b}{8}\right)\right|+2\left|f^{\prime}\left(\frac{a+b}{2}\right)\right|+\left|f^{\prime}\left(\frac{a+7 b}{8}\right)\right|\right]$.

(3) $x=\frac{a+b}{2}$, then we get

$$
\left|f\left(\frac{a+b}{2}\right)-\frac{1}{b-a} \int_{a}^{b} f(t) d t\right| \leq \frac{(b-a)}{4(1+p)^{1 / p}}\left[\left|f^{\prime}\left(\frac{3 a+b}{4}\right)\right|+\left|f^{\prime}\left(\frac{a+3 b}{4}\right)\right|\right] \text {. }
$$

3. A COMPOSITE QUADRATURE FORMULA

Let $I_{n}: a=x_{0}<x_{1}<\cdots<x_{n}=b$ be a division of the interval $[a, b]$ and $h_{i}=x_{i+1}-x_{i},(i=0,1,2, \cdots, n-1)$.

Consider the general quadrature formula

$$
Q_{n}\left(I_{n}, f\right):=\frac{1}{2} \sum_{i=0}^{n-1}\left[f\left(\frac{3 x_{i}+x_{i+1}}{4}\right)+f\left(\frac{x_{i}+3 x_{i+1}}{4}\right)\right] h_{i} .
$$

The following result holds.

Theorem 8. Let $f: I \subset \mathbb{R} \rightarrow \mathbb{R}$ be an absolutely continuous mapping on $I^{\circ}$, where $a, b \in I$ with $a<b$, such that $f^{\prime} \in L_{1}[a, b]$. If $\left|f^{\prime}\right|$ is convex on $[a, b]$. Then, we have

$$
\int_{a}^{b} f(t) d t=Q_{n}\left(I_{n}, f\right)+R_{n}\left(I_{n}, f\right) .
$$

where, $Q_{n}\left(I_{n}, f\right)$ is defined by formula (3.1), and the remainder term $R_{n}\left(I_{n}, f\right)$ satisfies the error estimates

$$
\begin{array}{r}
\left|R_{n}\left(I_{n}, f\right)\right| \leq \frac{1}{96} \sum_{i=0}^{n-1} h_{i}^{2}\left[\left|f^{\prime}\left(x_{i}\right)\right|+5\left|f^{\prime}\left(\frac{3 x_{i}+x_{i+1}}{4}\right)\right|\right. \\
\left.+5\left|f^{\prime}\left(\frac{x_{i}+3 x_{i+1}}{4}\right)\right|+\left|f^{\prime}\left(x_{i+1}\right)\right|\right] .
\end{array}
$$

Proof. Applying inequality (3.1) and (3.2) on the intervals $\left[x_{i}, x_{i+1}\right]$, we may state that

$$
R_{i}\left(I_{i}, f\right)=\int_{x_{i}}^{x_{i+1}} f(t) d t-\frac{1}{2}\left[f\left(\frac{3 x_{i}+x_{i+1}}{4}\right)+f\left(\frac{x_{i}+3 x_{i+1}}{4}\right)\right] h_{i} .
$$

Summing the above inequality over $i$ from 0 to $n-1$, we get

$$
\begin{aligned}
R_{n}\left(I_{n}, f\right) & =\sum_{i=0}^{n-1} \int_{x_{i}}^{x_{i+1}} f(t) d t-\frac{1}{2} \sum_{i=0}^{n-1}\left[f\left(\frac{3 x_{i}+x_{i+1}}{4}\right)+f\left(\frac{x_{i}+3 x_{i+1}}{4}\right)\right] h_{i} \\
& =\int_{a}^{b} f(t) d t-\frac{1}{2} \sum_{i=0}^{n-1}\left[f\left(\frac{3 x_{i}+x_{i+1}}{4}\right)+f\left(\frac{x_{i}+3 x_{i+1}}{4}\right)\right] h_{i},
\end{aligned}
$$


which follows from (2.2), that

$$
\begin{gathered}
\left|R_{n}\left(I_{n}, f\right)\right|=\left|\int_{a}^{b} f(t) d t-\frac{1}{2} \sum_{i=0}^{n-1}\left[f\left(\frac{3 x_{i}+x_{i+1}}{4}\right)+f\left(\frac{x_{i}+3 x_{i+1}}{4}\right)\right] h_{i}\right| \\
\leq \frac{1}{96} \sum_{i=0}^{n-1} h_{i}^{2}\left[\left|f^{\prime}\left(x_{i}\right)\right|+5\left|f^{\prime}\left(\frac{3 x_{i}+x_{i+1}}{4}\right)\right|\right. \\
\left.+5\left|f^{\prime}\left(\frac{x_{i}+3 x_{i+1}}{4}\right)\right|+\left|f^{\prime}\left(x_{i+1}\right)\right|\right] .
\end{gathered}
$$

which completes the proof.

Remark 2. One may state more inequalities, using (2.7) and (2.10). We shall omit the details.

\section{APPLICATIONS FOR P.D.F.'s}

Let $\mathrm{X}$ be a random variable taking values in the finite interval $[a, b]$, with the probability density function $f:[a, b] \rightarrow[0,1]$ with the cumulative distribution function $F(x)=\operatorname{Pr}(X \leq x)=\int_{a}^{b} f(t) d t$.

Theorem 9. With the assumptions of Theorem 5, we have the inequality

$$
\begin{aligned}
& \left|\frac{1}{2}[F(x)+F(a+b-x)]-\frac{b-E(X)}{b-a}\right| \\
& \leq \frac{(x-a)^{2}}{6(b-a)}\left(\left|F^{\prime}(a)\right|+\left|F^{\prime}(b)\right|\right) \\
& \quad+\frac{8(x-a)^{2}+3(a+b-2 x)^{2}}{24(b-a)}\left(\left|F^{\prime}(x)\right|+\left|F^{\prime}(a+b-x)\right|\right)
\end{aligned}
$$

for all $x \in\left[a, \frac{a+b}{2}\right]$, where $E(X)$ is the expectation of $X$.

Proof. In the proof of Theorem 5 , let $f=F$, and taking into account that

$$
E(X)=\int_{a}^{b} t d F(t)=b-\int_{a}^{b} F(t) d t
$$

We left the details to the interested reader.

Corollary 7. In Theorem 9, if we choose $x=\frac{3 a+b}{4}$, then we get

$$
\begin{aligned}
& \left|\frac{1}{2}\left[F\left(\frac{3 a+b}{4}\right)+F\left(\frac{a+3 b}{4}\right)\right]-\frac{b-E(X)}{b-a}\right| \\
& \leq \frac{(b-a)}{96}\left[\left|F^{\prime}(a)\right|+5\left|F^{\prime}\left(\frac{3 a+b}{4}\right)\right|+5\left|F^{\prime}\left(\frac{a+3 b}{4}\right)\right|+\left|F^{\prime}(b)\right|\right] .
\end{aligned}
$$


Corollary 8. In Theorem 9, if $F$ is symmetric about the $x$-axis, i.e., $F(a+b-x)=$ $F(x)$, we have

$$
\begin{aligned}
& \left|F(x)-\frac{b-E(X)}{b-a}\right| \\
& \leq \frac{(x-a)^{2}}{6(b-a)}\left(\left|F^{\prime}(a)\right|+\left|F^{\prime}(b)\right|\right) \\
& \quad+\frac{8(x-a)^{2}+3(a+b-2 x)^{2}}{24(b-a)}\left(\left|F^{\prime}(x)\right|+\left|F^{\prime}(a+b-x)\right|\right)
\end{aligned}
$$

for all $x \in\left[a, \frac{a+b}{2}\right]$.

Remark 3. One may state more inequalities, using Theorem 6 and Theorem 7. We shall omit the details.

\section{APPLICATIONS FOR SPECIAL MEANS}

Recall the following means which could be considered extensions of arithmetic, logarithmic and generalized logarithmic for positive real numbers.

(1) The arithmetic mean:

$$
A=A(a, b)=\frac{a+b}{2} ; a, b \in \mathbb{R}^{+}
$$

(2) The logarithmic mean:

$$
L(a, b)=\frac{b-a}{\ln |b|-\ln |a|} ;|a| \neq|b|, a, b \in \mathbb{R}^{+}
$$

(3) The generalized logarithmic mean:

$$
L_{n}(a, b)=\left[\frac{b^{n+1}-a^{n+1}}{(b-a)(n+1)}\right]^{\frac{1}{n}} ; n \in \mathbb{Z} \backslash\{-1,0\}, a, b \in \mathbb{R}^{+}, a \neq b
$$

(4) The identric mean:

$$
I(a, b)=\left\{\begin{array}{cl}
\frac{1}{e}\left(\frac{b^{b}}{a^{a}}\right)^{\frac{1}{b-a}} & , a \neq b \\
a & , a=b
\end{array} a, b \in \mathbb{R}^{+} .\right.
$$

Now using our results, we give some applications to special means for positive real numbers.

Proposition 1. Let $a, b \in \mathbb{R}^{+}, a<b$. Then, we have

$$
\left|A\left(\frac{4}{3 a+b}, \frac{4}{a+3 b}\right)-L^{-1}(a, b)\right| \leq \frac{(b-a)}{96}\left[\frac{a^{2}+b^{2}}{a^{2} b^{2}}+80\left(\frac{1}{(3 a+b)^{2}}+\frac{1}{(a+3 b)^{2}}\right)\right] .
$$

Proof. The assertion was obtained by the inequality in (2.2) applied to the convex mapping $f:[a, b] \rightarrow \mathbb{R}, f(x)=\frac{1}{x}$. 
Proposition 2. Let $a, b \in \mathbb{R}^{+}, a<b$, and $p \in \mathbb{Z},|p| \geq 2$.Then,

$$
\left|A^{p}(a, b)-L_{p}^{p}(a, b)\right| \leq \frac{p(b-a)}{24}\left[a^{p-1}+4 A^{p-1}(a, b)+b^{p-1}\right] .
$$

Proof. The assertion was obtained by the inequality in (2.3) applied to the convex mapping $f:[a, b] \rightarrow \mathbb{R}, f(x)=x^{p}$.

Proposition 3. Let $a, b \in \mathbb{R}^{+}, a<b$. Then, we have

$$
|\ln I-A(\ln a, \ln b)| \leq \frac{(b-a)}{2^{1+\frac{1}{q}}(1+p)^{\frac{1}{p}}}\left(\frac{1}{a^{q}}+\frac{1}{b^{q}}\right)^{\frac{1}{q}} .
$$

Proof. The assertion was obtained by the inequality in (2.6) applied to the convex mapping $f:[a, b] \rightarrow[0, \infty), f(x)=-\ln x$.

\section{REFERENCES}

[1] M. Alomari and M. Darus, "Some Ostrowski type inequalities for quasi-convex functions with applications to special means," RGMIA Preprint, vol. 13, no. 2, p. article No. 3., 2010.

[2] M. Alomari, M. Darus, S. S. Dragomir, and P. Cerone, "Ostrowski type inequalities for functions whose derivatives are $s$-convex in the second sense," Appl. Math. Lett., vol. 23, no. 9, pp. 10711076, 2010.

[3] M. W. Alomari, "A companion of Ostrowski 's inequality with applications," Transylv. J. Math. Mech., vol. 3, no. 1, pp. 9-14, 2011.

[4] N. S. Barnett, S. S. Dragomir, and I. Gomm, "A companion for the Ostrowski and the generalised trapezoid inequalities," Math. Comput. Modelling, vol. 50, no. 1-2, pp. 179-187, 2009.

[5] S. S. Dragomir, "A companion of Ostrowski's inequality for functions of bounded variation and applications," RGMIA Preprint, vol. 5, p. article No. 28, 2002.

[6] S. S. Dragomir, "Some companions of Ostrowski's inequality for absolutely continuous functions and applications," Bull. Korean Math. Soc., vol. 42, no. 2, pp. 213-230, 2005.

[7] S. S. Dragomir and R. P. Agarwal, "Two inequalities for differentiable mappings and applications to special means of real numbers and to trapezoidal formula," Appl. Math. Lett., vol. 11, no. 5, pp. 91-95, 1998.

[8] S. S. Dragomir and T. M. Rassias, Eds., Ostrowski type inequalities and applications in numerical integration. Dordrecht: Kluwer Academic Publishers, 2002.

[9] A. Guessab and G. Schmeisser, "Sharp integral inequalities of the Hermite-Hadamard type," J. Approximation Theory, vol. 115, no. 2, pp. 260-288, 2002.

[10] Z. Liu, "Some companions of an Ostrowski type inequality and applications," JIPAM, J. Inequal. Pure Appl. Math., vol. 10, no. 2, p. 12, 2009.

\section{Authors' addresses}

\section{Mohammad W. Alomari}

Department of Mathematics, Faculty of Science, Jerash University, Jerash, Jordan

E-mail address: mwomathegmail.com 
M. Emin Özdemir

Ağrı İbrahim Çeçen University, Faculty of Science and Letters, Department of Mathematics, 04100, Ağrı, Turkey

E-mail address: emoseatauni.edu.tr

Havva Kavurmac

Ağrı İbrahim Çeçen University, Faculty of Science and Letters, Department of Mathematics, 04100, Ağrı, Turkey

E-mail address: hkavurmacieatauni.edu.tr 